\title{
Methodological Issues in Facilitating Rhythmic Play with Robots
}

\author{
Marek P. Michalowski \\ Robotics Institute \\ Carnegie Mellon University \\ Pittsburgh, PA 15213, USA \\ michalowski@cmu. edu
}

\author{
Hideki Kozima \\ National Institute of Information \\ and Communications Technology \\ Kyoto 619-0289, Japan \\ xkozima@nict.go.jp
}

\begin{abstract}
We are currently investigating the use of rhythm and synchrony in human-robot interaction. Specifically, we are developing techniques for the perception and generation of social rhythmic behaviors in nonverbal dance-oriented play between children and a small creature-like robot. While our goal is to develop and evaluate technological artifacts that can participate in rhythmically coordinated social interactions, it is first necessary to develop and evaluate appropriate methodologies for the evaluation of such technology. In this paper we present our experience in developing and revising an experimental paradigm for the evaluation of dance-oriented play with a robot. We propose that strictly controlled experimental paradigms for even such constrained interactions preclude the full expression (and therefore observation) of interactive behaviors that can be considered sufficiently rich and natural for the study of the complex yet fundamental rhythmic properties of social interaction.
\end{abstract}

\section{INTRODUCTION}

The success of face-to-face human-robot social interaction depends strongly on the robot perceiving spatial and temporal properties of the interaction and behaving in a coordinated manner. Some easily observable aspects of this coordination, such as turn-taking, gestures, and emotive expression, are all important for effective communication and are popular areas of research in human-robot interaction. However, the comfort and "naturalness" of an interaction may also depend on more subtle and fine-grained coordination between the sounds and movements of the two interactors. Specifically, imitation and pattern are recognized in anthropology and social science literature as the foundation of a "dance" of coordinated sound and movement between interactors.

What we actually call dancing, usually to the accompaniment of music, in general consists of more structured, regular, and exaggerated behavioral rhythms than those observed in social interactions. It may therefore be a fruitful area in which to develop and test systems that would allow social robots to perceive and exhibit rhythmic behaviors and to participate in an interactive process of coordination and synchrony. Dance can also be an engaging and interesting component of playful interaction between robots and children, which is already a popular focus of human-robot interaction research for the purposes of pedagogy, cognitive development, and therapy. We believe that investigating the effects of rhythmic synchrony in dance-oriented humanrobot social interactions will be informative in designing robots that can perceive and behave according to natural rhythms in more open-ended interactions. However, while

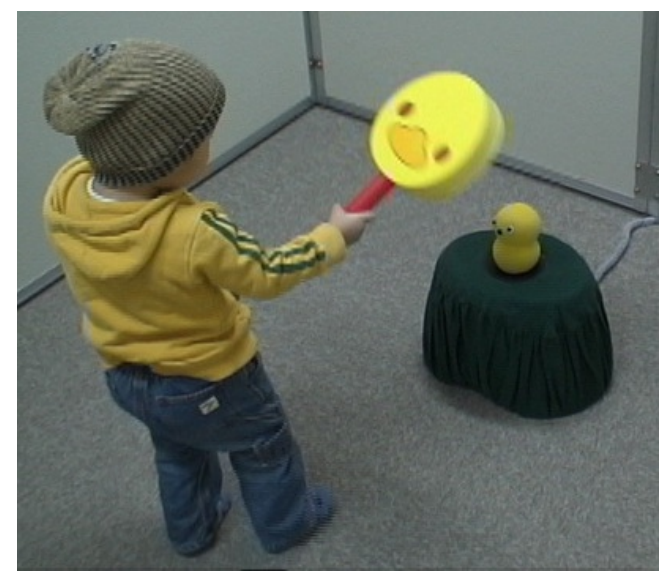

Fig. 1. Keepon dancing with a child.

our goal is to develop and evaluate technological artifacts that can participate in rhythmically coordinated social interactions, it is first necessary to consider appropriate methodologies for the evaluation of such technology.

Modern psychological experiments, in order to minimize the number of confounding factors, typically attempt to control differences between participants' experiences and to clearly define these differences in terms of independent or dependent variables. However, rhythmicity is a holistic property of social interaction, and a reductionist approach that attempts to cut this phenomenon into pieces (to see the effects of changes in one set of parameters on another set of parameters) looks only at the tip of an iceberg. Furthermore, enforcing a scenario that emphasizes a small number of piecewise relationships may actually stifle the holistic emergence of a phenomenon like social rhythmicity. While we started with an attempt to examine rhythmicity in a controlled manner, our experience leads us to re-evaluate our approach.

After reviewing background work in this area and describing the system we have designed, we present a description of a pilot study on the effects of synchrony with environmental and social rhythms on dance-oriented play between children and a robot (fig. 1). This study was intended to evaluate and improve our system, to observe a range of behaviors that we might expect in social interactions with such a system, and to develop an experimental paradigm appropriate for research in this important area. We believe that a looser, more naturalistic environment (and therefore a less strictly 
adhered-to experimental procedure) is necessary to promote rich interactions that can yield useful and interesting data for studying social rhythmic phenomena. Individual differences between children require that interactions under study incorporate adequate facilitation and that they be allowed to develop and unfold in a dynamic and flexible way.

\section{BACKGROUND}

The past four decades have witnessed the emergence and development of a line of research spanning disciplines such as anthropology, psychology, and cognitive science in understanding the organizing principles for interaction between intelligent embodied agents. We now understand interaction not as a sequential process of give-and-take, but as a dynamic process of coordinated activity between constantly adapting participants. Together with behavior matching (or posture mirroring, the correspondence between positions or gestures assumed by two interactors [19], [15]), interactional synchrony provides a foundation for interpersonal coordination [2] and emotional contagion [12]. Interactional synchrony is the temporal coordination of communicative behaviors between interactors (often without awareness or volition) in order to achieve a sort of "goodness of fit" between them [3].

The rhythmic organization of social interaction is an expression of the oscillatory neurobiological language of the central nervous system through learned cultural patterns [4]. Two or more people coordinate their rhythms, achieving synchrony, through a process known as entrainment [6], [14], whereby multiple different rhythms converge on or capture each other. Given the important role of rhythmicity and synchrony in human interaction, it is clear that difficulties in establishing interactional synchrony can make face-toface interpersonal communication and interaction difficult, if not impossible; in fact, marked asynchronies within an individual's own behaviors [7], along with abnormal entrainment in interpersonal interactions [5], are often characteristic of pathologies such as autism and schizophrenia. Rhythm, specifically in the forms of music and dance, is also an important part of human education across cultures; it is proposed that there is an inherent musicality in our social drive [21]. Dance has, in turn, been identified and used in therapies for a wide range of disorders [11], [9]. For our purposes, we can consider interactive dance-oriented play with a robot as an activity that can be used by clinicians to study a child's behavioral patterns and to identify rhythmic abnormalities that might be useful in diagnosing and perhaps even treating certain pathologies.

Attention to the rhythmic characteristics of nonverbal interaction has not been widely adopted in social robotics research. General ideas of turn-taking in conversation are widely implemented, but fine-grained rhythmic perception and synchrony by a robot has been difficult to develop. Ogawa et al. [18] recognize the importance of rhythmic entrainment to non-verbal cues such as nodding and gesture in vocal communcation. In their InterRobot humanoids, which are used in pairs for embodied telecommunication between remote interactors, they use nonverbal rhythmic cues such as facial expressions and bodily movements that are automatically synchronized to the speech input of a remote human interactor. Andry et al. [1], with the view of interpersonal coordination as a method of learning, use synchronized imitation as a way for robots to learn sensorymotor correspondences and rhythmic motion sequences. The humanoid robots Nico [8] and Haile [22] perform drumming synchronized to another person or a conductor as an example of the kind of rhythmic entrainment that might be applied to other social tasks. In existing projects, rhythmic interaction is generally based on auditory cues and limited perception of embodied movement. However, robots should be able to "tune in" to the bodily rhythms of their interaction partners as well as to generate such nonverbal behaviors (e.g., nodding, swaying, gesturing) themselves.

Tanaka et al. [20] created non-interactive and interactive (posture mirroring) dance modes for a QRIO robot in a playroom with children. They found a significant difference between the two conditions with respect to the time children spent with the robot, yet this experiment concerned contingency rather than the type of rhythmic synchrony we are developing. We have observed similar differences between contingent and non-contingent behaviors in rhythmic interaction between children and a robot-like puppet [17], and furthermore observed a higher incidence of rhythmic play by children when the robot behaved contingently. Based on exploratory observations of the herein described system [16], we believe that the robot's synchrony with environmental and social rhythms has a strong effect on the quality of resulting social interactions.

Since both self-synchrony (rhythmic matching of movements and vocalizations) and interactional synchrony are important for normal human-human interaction [13], [7], these capabilities should be considered in designing any robots that interact socially with humans, not only those that are designed for therapeutic or educational interactions with children. We now present our system and our development and evaluation of an experimental paradigm for this line of research.

\section{SySTEM DESIGN}

\section{A. Hardware}

The creature-like robot Keepon is designed to perform emotional and attentional communication with human interactors, especially children. Keepon has a snowman-like body (fig. 2). The upper part (the "head") has two eyes (each of which is a $120^{\circ}$ wide-angle color CCD camera) and a nose (which is a microphone). The lower part (the "belly") has a small gimbal and four wires by which the body is manipulated like a marionette. Keepon sits atop a black cylinder that contains four motors and two circuit boards (a PID controller and a motor driver). Since Keepon's body is made of silicone rubber and is relatively hollow, the head and belly deform whenever Keepon changes posture or when someone touches it.

The simple body has four degrees of freedom (DOFs), as shown in fig. 3: nodding/tilting $\pm 40^{\circ}$, shaking/panning $\pm 180^{\circ}$, rocking side-to-side $\pm 25^{\circ}$, and bobbing/shrinking 


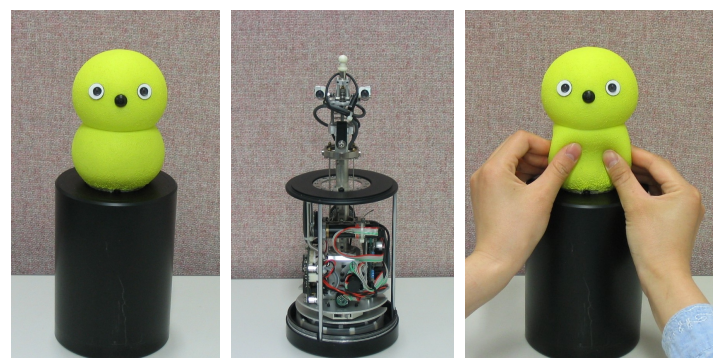

Fig. 2. Keepon's external and internal structure and its deformable body.
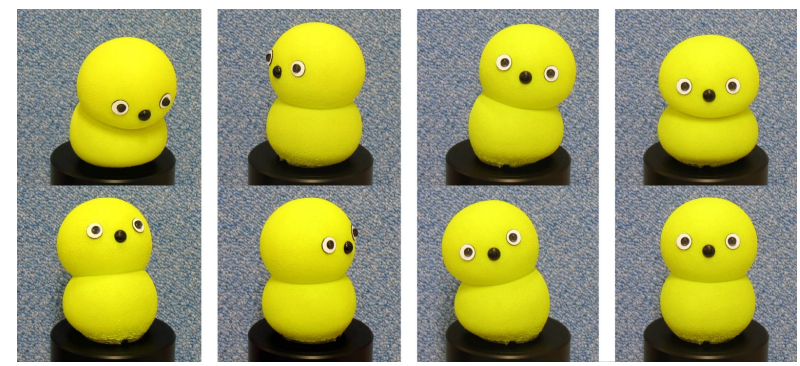

Fig. 3. Keepon's four degrees of freedom (nodding, panning, rocking, and bobbing).

with a $15 \mathrm{~mm}$ stroke. For each degree of freedom, the PID controller can be given parameters for maximum velocity and acceleration. Given a position command, the controller generates a trapezoidal velocity profile that smoothly accelerates and decelerates the motor to the desired position.

\section{B. Software}

A number of low-level software modules on the controlling computer are used for command generation and serial communication with the robot. High-level control is implemented in Max/MSP [10], a graphical programming environment originally designed for controlling digital musical instruments. Max/MSP is well-suited to this application, as it provides tools and components for creating clocks and metronomes, performing audio signal processing, and designing graphical user interfaces.

An interface built in Max/MSP allows a human teleoperator to see the view from Keepon's eyes and to control the robot's pose or direction of attention. While it would be possible to run the robot completely autonomously using, for example, face recognition, having a human control the robot's high-level attentional state allows for more compelling social interactions while focusing on the relationship between the autonomous rhythmic behaviors of robot and interactor.

A metronome in Max/MSP produces a succession of beats separated by a given time interval to produce the desired beats per minute (BPM). The frequency of the master beat to which Keepon dances can be obtained from multiple sensory modalities (in [16] we used visual sensing, computing average optical flow in a region of interest; in this work, we use an accelerometer, described below). The Max/MSP sync ${ }^{\sim}$ object receives a stream of beats and produces an oscillator that is synchronized with the tempo.

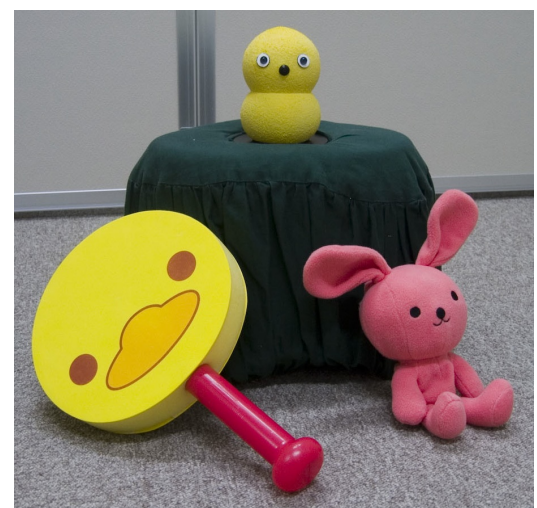

Fig. 4. Keepon and the two toys used with the accelerometer: a stuffed rabbit $(169 \mathrm{~g}, 290 \mathrm{~mm}$ long) and a soft paddle $(137 \mathrm{~g}, 320 \mathrm{~mm}$ long).

This oscillator drives a stream of commands that cyclically move Keepon's bobbing and rocking degrees of freedom; nodding and panning are used by the teleoperator to focus Keepon's attention on a particular location. The dancing mechanism is described in more detail in [16].

Finally, a sequencer is used to record aligned streams of beats, sensor data, and motor commands (from the music, the accelerometer, and Keepon, respectively) for later playback and analysis.

\section{Accelerometer}

To overcome the difficulties of sensing human movement through vision as in [16], we have been developing an additional system for detecting rhythmic movement through the use of a battery-powered three-axis accelerometer, with wireless Bluetooth data transfer, implanted in a toy (fig. 4). We expected the use of a toy to carry a number of benefits:

- It might encourage more exaggerated, regular, repetitive movement, as seen in child's play with dolls (e.g. walking, talking);

- It might allow the child to use his or her imagination to create a narrative involving the toy and Keepon as characters; and

- The toy, being of similar size and form to Keepon, might be seen as the robot's "peer" through which the child might develop a different type of relationship with the robot.

The accelerometer provides force data for three axes of movement. The magnitude of the overall acceleration is the Euclidian norm of the vector defined by these three values. We detect rhythmic movements of the toy by finding peaks in this magnitude. Since the sensor data is rather noisy, these peaks, or direction change points, are found from zero-crossings in lowpass-filtered data. We can then treat these direction change points as "beats" in the same way as musical beats or visual movement direction changes were described above.

\section{PROPOSED EXPERIMENTAL DESIGN}

Our initial conception of the experimental procedure was designed to examine, in a controlled manner, the active or passive roles that a child and a robot might take in 
a rhythmic social interaction. We designed the described control architecture for such an experiment in order to allow the robot Keepon to synchronize its dancing behaviors to music or to the movement of a toy, to present stimuli in a controlled manner, and to record sensor data for later analysis and comparison with the rhythms of the musical stimuli and the robot's movements.

Our procedure was as follows: A facilitator brings the child and caregiver into a play area $(1.9 \mathrm{~m}$ by $3.12 \mathrm{~m}$, wall height $1.5 \mathrm{~m}$ ) with Keepon on the floor about $50 \mathrm{~cm}$ from the middle of one of the shorter walls. The facilitator retrieves a stuffed rabbit toy (equipped with a wireless accelerometer) from behind a wall of the play area, and Keepon establishes gaze following (with the guidance of a teleoperator) toward the toy. The facilitator demonstrates Keepon's contingency and maintenance of attention to the movement of the toy. The facilitator places the rabbit next to Keepon and Keepon gazes at it. As soon as the child picks up the toy, music begins to play and Keepon begins to dance. Keepon dances only when the child is holding and moving the toy (automatically sensed by the accelerometer). Meanwhile, the teleoperator maintains Keepon's attention toward the toy. The only difference between conditions is that, in the first condition, the rhythm of Keepon's dance is synchronized with that of the music; in the second condition, the rhythm is derived from the detection of direction change points in the movement of the accelerometer. After two songs (approximately $4 \mathrm{~min}$.), an indefinite period of free play is allowed, with rhythmic synchrony to the movement of the toy (for all children).

There are a few important points to note about this procedure:

- A teleoperator maintains Keepon's attention toward the toy for the duration of the experiment.

- During the presentation of musical stimuli, and during free play, Keepon's expression of rhythmic dancing behavior is in both conditions contingent on movement of the toy.

- There are (at least) three important rhythms in effect during this type of interaction: the environmental rhythm of the music, the rhythm of Keepon's dancing, and the rhythm of the child's movement of the toy. All three rhythms are recorded for later comparison and analysis.

Fig. 5 illustrates the proposed relationship between rhythms in the two conditions.

The recorded rhythm of the child's movement of the toy may be analyzed for quantitative information about three variables: first, the number of movements made by the child (i.e., in fig. 5, the child changes the toy's direction seven times); second, the duration of movement by the child (i.e., in fig. 5, the total number of seconds in the three periods of movement); third, the degree of synchrony between the child's movements and the music (i.e., the correspondence between tempos in the two rhythms). These values, in addition to the qualitative analysis of recorded video, can provide a numerical indication of the difference between experimental conditions.
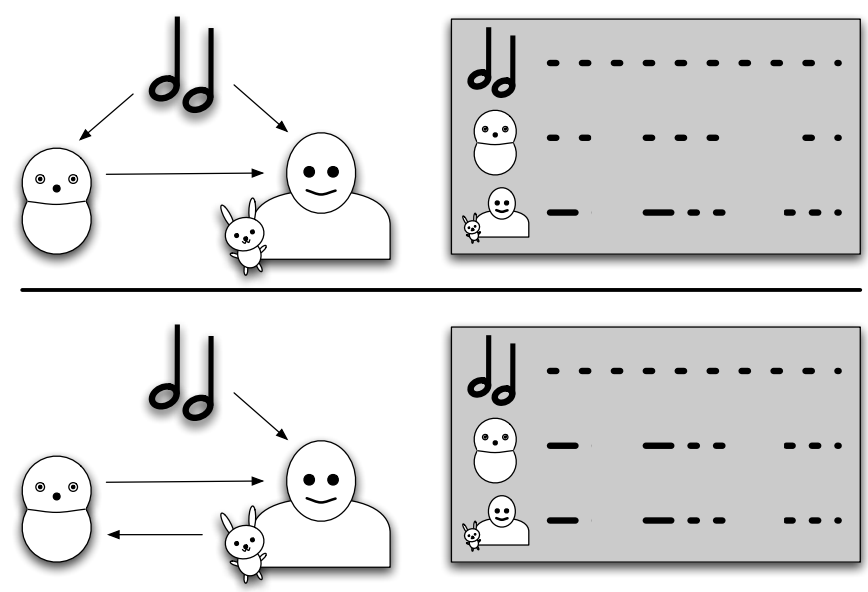

Fig. 5. The proposed "flow" of rhythmic influence between the music, the robot, and the child in the two experimental conditions: Keepon synchronizing to the music (top) and to the toy (bottom).

In the first condition, Keepon is synchronized with the music's rhythm; we might say that Keepon is following the environmental rhythm and trying to lead the child's rhythms. In the second condition, Keepon should follow or be synchronized with the child's rhythm. The goal of this experiment is to determine which condition results in a closer coordination between the three rhythms. If it is the first condition, it may suggest that Keepon's physical expression (and reinforcement) of audible environmental rhythms is salient and encourages the child to follow this rhythm as well. If it is the second condition, it may suggest that the child's perception of Keepon's rhythmic contingency to his or her own will encourage the child to more clearly and actively follow the environmental rhythms and to lead Keepon's rhythm so that the two may dance to the music together.

\section{CONDUCTING (AND REVISING) THE EXPERIMENT}

We recruited eight volunteer parents to bring their 3year-old children (34.69 \pm 0.736 months) to a child study laboratory at Kyushu University in Japan in February 2007. As the experiment was explained to parents and consent forms filled out, children played in a staging area with young lab assistants to make them feel comfortable. Next, the experimental sessions lasted about twenty minutes each. Finally, a debriefing session in the staging area was used to explain the system and the reasons for the study.

After three sessions, we found that our choice of toy had an impact on the sensor data we obtained. The shape and weight distribution of the toy was such that children tended to hold the rabbit by the neck and spin it rapidly back and forth by rotating their wrists. This movement was both difficult to sense (being rotation rather than translation around the center of the accelerometer) and too rapid to provide meaningful rhythmic input to the system. For the remaining sessions, we selected a different toy: a foam paddle with a plastic handle and the face of a yellow "chick"; this could plausibly be called Keepon's "mother" and provided an affordance for holding and waving rather 
than spinning. For four of the remaining five children, the difference with this toy was indeed the elicitation of a different quality of movement, and sensor data that reflected the clearer rhythmicity of this movement.

We also closely followed the experimental procedure described above for the first three sessions (Keepon synchronized to the toy for the first two sessions, and to the music for the third) and noted a common shyness or reluctance to engage with the robot, which is typically a very interesting artifact for children in the classroom settings in which we normally observe it. We suspected that the facilitator's limited introductory behaviors with the robot, and his subsequent withdrawal from the interaction (all intended for controllability), resulted in the lack of a scaffold or context for the children to begin their exploratory play. The short demonstration of Keepon's contingency to the location of the toy was hindered by a competition between the robot and the toy for the child's attention; since the robot was moving under its own power, children focused on it as a novel sort of artifact. This novelty meant nearly no engagement during the music, and generally only an exploration of contingency after some time during the postmusic free play period.

Therefore, we decided to introduce an introductory free play period before the music, with only attentional contingency, to remove some of the element of novelty and surprise during the music. For the fourth and fifth participants, the change appeared to result in a higher degree of comfort and uninhibited play during the musical period. However, we found that the introduction of music itself seemed to be a novel enough stimulus that it continued to elicit an initial period of uncertainty or renewed observation for any difference in the robot's reaction with the music, not necessarily providing the active movement of the toy necessary to contingently trigger Keepon's dancing. We also saw the fourth and fifth participants rhythmically hit Keepon's head with the paddle, and this was sufficiently reinforced by Keepon's responsive rhythmic behavior that it was repeated. However, this behavior was not performed with music or for sufficient duration to provide meaningful quantitative rhythmic data.

Therefore, we further modified the procedure to add music in the hopes that this exploratory behavior would, with more time, lead to desired rhythmic behavior with the music. After the introductory attention-contingent free play, a song was added in which the robot's rhythm was synchronized with that of the toy for all children. Following a thirtysecond pause, two songs played in which the robot danced in synchrony with the music. Following another pause, a fourth song played with toy-synchronized dancing once again. For the limited number of children, we decided that any differences between conditions were overwhelmed by individual differences between participants.

The sixth participant was extremely shy and unresponsive to almost any of the robot's behaviors or the facilitator's encouragement. The seventh and eighth participants, however, performed a high amount of rhythmic behavior, particularly after becoming comfortable with the robot's attentional contingency and the presence of music. For these last two participants, we further deviated from the procedure in making Keepon much more social, in the sense of frequently shifting attention between the child and the toy, and this seemed to be a powerful cue for motivating use of the toy. In the case of the eighth participant, a five-year-old brother was permitted to participate in the interaction, and we found that his company and facilitation encouraged exactly the kind of rhythmic behavior we had hoped to see, without seeming to bias or detract from the free expression of these behaviors or their synchrony with the robot or the music. Running around Keepon was seen by this final pair as the most interesting way of eliciting movement and attentional contingency. During one period, when the final participant actively moved the paddle for Keepon, we observed a mixture of attention-directing and rhythmic stimuli from the child: she made large movements of the paddle and held it in particular locations for Keepon to gaze at, interspersed with rapid repetitive waving of the paddle to elicit dancing. It is our opinion that she recognized that these were qualitatively different types of stimuli that elicited qualitatively different behaviors from the robot.

While we did not observe as much rhythmic behavior from most of the children as we had hoped, we are closer to an understanding of the types of scenarios that are conducive to such behavior.

\section{DISCUSSION}

A number of problems were responsible for the low rate of rhythmic engagement, and we believe that identifying these problems is valuable for human-robot interaction research involving children.

The first problem we face is a technical one: that of perceiving rhythmic behavior. We have been investigating the use of vision, sound, and an accelerometer in detecting social rhythms. Our system is designed to be amodal, such that a number of additional modalities are possible, e.g., placing the accelerometer on the body of the child, using motion capture systems, installing pressure pads in the floor for detecting gross vertical movement, etc. Meanwhile, the problem of building rhythmic synchrony goes beyond perception and behavior to the way in which social rhythms should be represented and acted upon. For example, we have observed that correspondence in tempo is not as effective or engaging as a matching of phase (or the co-occurence of beats). A purely reactive system cannot, theoretically, be synchronous in this way, due to delays in sensing, processing, and acting. It may be argued that one of the reasons for the rhythmic organization of social behavior is that it enables synchrony and simultaneous movement through anticipation. Interacting partners might be seen to have a responsibility to anticipate the behaviors of the other and to "respect" the other's anticipation of one's own behavior. While our system is still not anticipatory in this way, it will be possible to account for inherent delays and to compensate for them.

A second problem, specifically in the evaluation of our technology, is the wide range of possible interactive modes 
that children might exhibit. These differences might stem from prior exposure or pre-existing ideas about robots, styles of play at home, comfort around strangers, and so on. This unpredictability and variability make it difficult to create well-defined procedures and consistent scenarios between participants in such a study. The facilitator, as we have observed, should establish a sufficient context for social interaction between the child and the robot. To do this effectively, he or she must be allowed to revise or even craft (in real time, based on his or her observations) a customized procedure that accounts for the individual personality and disposition of the child.

A third problem is that the novelty of the robot, and the explorative behavior induced by this novelty (especially combined with the unfamiliar, sterile, anxiety-inducing setting of a typical laboratory and the artificial scenario), seem to overwhelm the free and natural expression of rhythmic behavior. For example, children are usually testing the robot's contingency or watching to see what it will do next, rather than assuming the basic social contingency they would expect in another human or in a pet. We expect that children familiar with Keepon would act very differently, and in a rhythmically more interesting manner, than did the children in this study. Although this prior exposure might be considered a bias, we believe that familiarity and comfort with the robot are actually necessary for the expression and observation of the behaviors in which we are interested.

Over the course of our observations, we have come to believe that children's motivation to explore is related to their perception of controllability or reactivity in the robot. The most basic form of this controllability is the recognition of contingency. In our experience, contingency in attention to a spatial location is the most salient form of contingency because it occurs instantaneously. On the other hand, rhythmic synchrony has temporal duration and requires sustained observation. Even when rhythmic contingency was recognized by children, it was usually recognized as contingency in the onset of oscillatory movement (and, to a lesser extent, as a general correlation in frequency) rather than in the establishment or maintenance of rhythmic synchrony. Therefore, it may be necessary to saturate the interest in instantaneous contingency in order to encourage children's attention to continuous behaviors. A flexible and accommodating interactive scenario, in addition to the promotion of familiarity with the social responsiveness of the robot, should allow us to observe and rigorously analyze the subtle properties of social rhythmic synchrony such as we were finally able to observe.

\section{ACKOWLEDGMENTS}

We would like to thank Kazuhide Hashiya, Hiromi Kobayashi, Saori Tanaka, and Kentaro Yamamoto (at Kyushu University) for their assistance and advice in conducting the described experiment. This work was supported by JSPS grant \#18330144 and a NSF Graduate Research Fellowship.

\section{REFERENCES}

[1] P. Andry, P. Gaussier, S. Moga, J. P. Banquet, and J. Nadel. Learning and communication via imitation: An autonomous robot perspective. IEEE Transactions on Systems, Man, and Cybernetics, 31(5), Sept 2001.

[2] F. Bernieri and R. Rosenthal. Interpersonal coordination: Behavior matching and interactional synchrony. In R. Feldman and B. Rime, editors, Fundamentals of nonverbal behavior, pages 401-432. Cambridge University Press, 1991.

[3] J. K. Burgoon, L. A. Stern, and L. Dillman. Interpersonal Adaptation: Dyadic Interaction Patterns. Cambridge University Press, New York, 1995.

[4] E. D. Chapple. Movement and sound: The musical language of body rhythms in interaction. In M. Davis, editor, Interaction Rhythms: Periodicity in Communicative Behavior, pages 31-51. Human Sciences Press, New York, 1982.

[5] W. S. Condon. Communication: Rhythm and structure. In J. R. Evans and M. Clynes, editors, Rhythms in Psychological, Linguistic and Musical Processes. Charles C. Thomas Publisher, Springfield, 1986.

[6] W. S. Condon and W. Ogston. Sound film analysis of normal and pathological behavior patterns. Journal of Nervous and Mental Disease, 143:338-347, 1966.

[7] W. S. Condon and L. W. Sander. Synchrony demonstrated between movements of the neonate and adult speech. Child Development, 45(2):456-462, June 1974.

[8] C. Crick, M. Munz, and B. Scassellati. Synchronization in social tasks: Robotic drumming. In Proceedings of the 15th IEEE International Symposium on Robot and Human Interactive Communication (RO-MAN 2006), University of Hertfordshire, United Kingdom, September 2006

[9] R. F. Cruz and C. Berrol, editors. Dancelmovement therapists in action: A working guide to research options. Charles C. Thomas, Springfield, IL, 2004.

[10] Cycling ' 74 . Cycling ' 74 Max/MSP: A graphical programming environment for music, audio, and multimedia., 2006. URL: http://www.cycling74.com/products/maxmsp.

[11] H. Fledderjohn and J. Sewickley. An Annotated Bibliography of Dance/Movement Therapy, 1940-1990. American Dance Therapy Association, Columbia, MD, 1993.

[12] E. Hatfield, J. T. Cacioppo, and R. L. Rapson. Emotional Contagion. Cambridge University Press, New York, 1994.

[13] A. Kendon. Movement coordination in social interaction: Some examples described. Acta Psychologica, 32:100-125, 1970.

[14] A. Kendon. Conducting Interaction: Patterns of behavior in focused encounters. Cambridge University Press, 1990.

[15] M. LaFrance. Nonverbal synchrony and rapport: Analysis by the cross-lag panel technique. Social Psychology Quarterly, 42(1):6670, March 1979.

[16] M. P. Michalowski, S. Sabanovic, and H. Kozima. A dancing robot for rhythmic social interaction. In Proceedings of the 2nd Annual Conference on Human-Robot Interaction (HRI 2007), pages 89-96, Washington, DC, March 2007. ACM.

[17] M. P. Michalowski, S. Sabanovic, and P. Michel. Roillo: Creating a social robot for playrooms. In Proceedings of the 15th IEEE International Symposium on Robot and Human Interactive Communication (RO-MAN 2006), pages 587-592, University of Hertfordshire, UK, September 2006. IEEE.

[18] H. Ogawa and T. Watanabe. Interrobot: speech-driven embodied interaction robot. Advanced Robotics, 15:371-377, 2001.

[19] A. Scheflen. The significance of posture in communication systems. Psychiatry, 27:316-331, 1964.

[20] F. Tanaka, J. R. Movellan, B. Fortenberry, and K. Aisaka. Daily hri evaluation at a classroom environment: Reports from dance interaction experiments. In Proceedings of the 1st Annual Conference on Human-Robot Interaction, pages 3-9, Salt Lake City, Utah, 2006.

[21] C. Trevarthen and S. N. Malloch. The dance of wellbeing: Defining the musical therapeutic effect. Nordic Journal of Music Therapy, 9:3-17, 2000.

[22] G. Weinberg and S. Driscoll. Robot-human interaction with an anthropomorphic percussionist. In Proceedings of CHI2OO6, pages 1229-1232, Montreal, Canada, 2006. 